\title{
Shear Bond Strength of the Metal Bracket to Zirconium Ceramic Restoration Treated by the Nd: YAG Laser and Other Methods: An In Vitro Microscopic Study
}

\author{
Hadi Mokhtarpur ${ }^{1 \#}$, Maliheh Nafisifard ${ }^{2 \#}$, Sepideh Dadgar ${ }^{3 \#}$, Ardavan Etemadi $^{4}$, Nasim Chiniforush ${ }^{5,6^{*}}$, Farhad \\ Sobouti ${ }^{3,7^{*}}$ \\ ${ }^{1}$ Prosthodontic Department, Dental Faculty, Mazandaran University of Medical Sciences, Sari, Iran \\ ${ }^{2}$ Restorative Dentistry Department, Dental Faculty, Babol University of Medical Sciences, Babol, Iran \\ ${ }^{3}$ Orthodontic Department, Dental Faculty, Mazandaran University of Medical Sciences, Sari, Iran \\ ${ }^{4}$ Department of Periodontics, Faculty of Dentistry, Tehran Medical Sciences, Islamic Azad University, Tehran, Iran \\ ${ }^{5}$ Dental Implant Research Center, Dental Research Institute, Tehran University of Medical Sciences, Tehran, Iran \\ ${ }^{6}$ Department of Surgical Sciences and Integrated Diagnostics, University of Genoa, Italy \\ ${ }^{7}$ Dental Sciences Research Center, Mazandaran University of Medical Sciences, Sari, Iran
}

\# These authors contributed equally to the work.

\section{*Correspondence to} Farhad Sobouti, DDS, MSc, Orthodontic Department, Dental Faculty, Mazandaran University of Medical Sciences, Sari, Iran. Email: farhad_sobouti@ yahoo.com; Nasim Chiniforush, DDS, PhD, Assistant Professor, Dental Implant Research Center, Dentistry Research Institute, Tehran University of Medical Sciences, Tehran, Iran. Tel/ Fax: +98-2183384000; Email: n-chiniforush@sina.tums.ac.ir

Published online October 3 2020

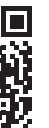

\begin{abstract}
Introduction: Providing reliable bonding of the bracket base and the zirconia surface is required to apply orthodontic force. The purpose of this scientific experiment was to evaluate the efficacy of three different methods of surface preparation for Zirconia, including surface roughening, sandblasting and the Nd: YAG laser, in the shear bond strength (SBS) of the orthodontic brackets. Methods: Fifty-four discs of zirconia were divided into three groups of 18: A) Hydrofluoric acid etching, B) sandblasting, and C) $\mathrm{Nd}$ : irradiation using the power of $1.5 \mathrm{~W}$ for 10 seconds. After bonding the brackets, the samples were slowly thermo-cycled (1000 times) for 24 hours. The SBS test was performed by a universal testing machine at a head speed of $0.5 \mathrm{~mm} / \mathrm{min}$. The adhesive remnant index (ARI) was scored at a magnification of 10 in the stereo microscope. All data were collected and analyzed using the variance, Kruskal-Wallis, Tukey, Don, and Weibull tests $(\alpha=0.05)$. Results: The HF acid etching group $(6.11 \pm 0.94 \mathrm{MPa})$ had the highest SBS, which was followed by the laser group $(6 \pm 0.61 \mathrm{MPa})$ and the sandblast group $(3.1080 \pm 0.82 \mathrm{MPa})$. There was a significant statistical difference between the laser and HF groups and the sandblast group $(P<0.05)$ and no significant difference between the HF group and the laser group $(P=0.03)$.

Conclusion: Based on the obtained bond strength, the Nd: YAG laser with a power of $1.5 \mathrm{~W}$ could be a substitute treatment method for the HF acid-etching.

Keywords: Hydrofluoric acid; Laser; Shear strength; Zirconium.
\end{abstract}

\section{Introduction}

The demand for a beautiful and attractive smile stimulates many adult patients to seek orthodontic treatments. Many of these patients have resin composite restorations, amalgam, gold and acrylic resin or porcelain restorations in their mouths. ${ }^{1,2}$ A large number of all ceramic restorations are made of zirconium. These crowns are popular and widely used because of their benefits such as biocompatibility, beauty, high resistance to breakage and accuracy in manufacturing. ${ }^{3}$ As already said, providing reliable bonding between the bracket and the surface of zirconia is necessary for orthodontic forces. This connection should be strong enough to prevent bonding failure during orthodontic treatment and to protect the integrity of zirconia during the deboning of brackets at the end of orthodontic treatment. Since the past, bonding orthodontic brackets to ceramic surfaces has been a challenge to orthodontists. ${ }^{4-7}$ One of the most commonly used substances for all ceramic crowns is Zirconium.

Due to the frequent report of veneer fracture in the posterior teeth, which is caused by intense masticator force, ${ }^{7,8}$ it is widely encouraged to use monolithic zirconium crowns without veneers. ${ }^{9,10}$

When monolithic zirconium crowns are used, the orthodontic bracket is immediately bonded to the underlying zirconium surface.

Since thorough roughening can cause micro-cracks that undermine the overall integrity of the ceramic surface,

Please cite this article as follows: Mokhtarpur H, Nafisifard M, Dadgar S, Etemadi A, Chiniforush N, Sobouti F. Shear bond strength of the metal bracket to zirconium ceramic restoration treated by the Nd: YAG laser and other methods: an in vitro microscopic study. J Lasers Med Sci. 2020;11(4):411-416. doi:10.34172/jlms.2020.65. 
bonding methods that provide more strength while using less roughening are recommended. ${ }^{11-13}$

The use of hydrofluoric acid (9/6\%) for 2 to 3 minutes can be described as a standard technique that provides sufficient bond strength for bracket-ceramic connection $^{12-14}$; however, it has the disadvantage of producing toxic vapors, skin burns, and mucous membrane injury. In addition, it damages the surface of zirconia. Thus, an alternative method which causes less soft tissue and zirconia damage is sought after., ${ }^{2,5}$ Other alternatives for the preparation of the ceramic surfaces prior to the orthodontic bracket bonding include surface roughening with a diamond bur or sandblasting and the use of phosphoric acid gel (37\%), but the resistance of the bond is clinically insufficient. ${ }^{15,16}$

The use of different kinds of lasers as a replacement option in these treatments has been proposed with convenient results. The Nd:YAG laser can be used for porcelain conditioning by creating surface roughness through melting and recrystallization. Since many clinicians and authors believe laser irradiation surpasses other methods in preparing the enamel and porcelain surfaces, ${ }^{17}$ the aim of this study is to compare the efficacy of the three widely used methods of zirconium surface treatment, including 9.6\% HF acid etching, Nd: YAG laser irradiation and sandblasting, in the shear bond strength (SBS) of metal brackets.

\section{Materials and Methods}

Fifty-four Yttria Tetragonal Zirconia Polycrystal (Y-TZP) (Cercon 1, DeguDent, Hanau, Germany) samples with 8 $\mathrm{mm}$ diameter and $4 \mathrm{~mm}$ height were used for this in vitro study. The samples were mounted on a self-curing acrylic resin using a plastic cylindrical mold.

We randomly divided Zirconia samples into three groups (n: 18). A $0.8 \mathrm{~mm}$ round diamond bur was used to remove the glazed layer of the zirconium disk.

Group a: 9.6\% HF acid (Pulpdent, Watertown, USA) was used for 3 minutes to etch the zirconia disk surface that was then washed off for 15 seconds using water with a gentle flow and later we used a blower for 15 seconds to dry the surface.

Group b: In this group, Nd:YAG laser irradiation (Fotona, Slovenia) was used to prepare the zirconia surface. The samples were exposed to the output power of $1.5 \mathrm{~W}$, Energy of $150 \mathrm{~mJ}$ energy, frequency of $10 \mathrm{~Hz}$, and pulse duration of $100 \mu \mathrm{s}$. The irradiation time was 40 seconds and the fiber diameter used was $320 \mu \mathrm{m}$. Laser movement was done by hand and in a linear pattern. The power density of the beam was $1866.04 \mathrm{~W} / \mathrm{cm}^{2}$.

Group c: Sandblasting (Renfert, Hilzingen, Germany) with aluminum oxide particles of $110 \mu \mathrm{m}$, at 80 Psi pressure was used for 4 seconds.

Silane primer (Pulpdent, Watertown, USA) was smeared on the surface of the zirconia and then dried following the initial preparing procedures.
Pre-adjusted 0.022 inches, stainless steel maxillary central incisor brackets (Dentsply Gac, NY, USA) were used in this study.

A composite bonding system, comprising two components of primer and adhesive, was used by the same operator to bond all the brackets in this study. (Transbond XT, 3M/Unitek, Monrovia, CA, USA). A light-emitting diode (Ledition, Ivoclar Vivadent AG, Schaan, Lichtenstein) was used to light cure the adhesive for 40 seconds. The materials were put in a $37^{\circ} \mathrm{C}$ water bath for 24 hours after polymerization. The samples were subjected to 1000 thermal cycles of 5 to $55^{\circ} \mathrm{C}$ for 30 seconds with 10 -second transfer time. After we perpendicularly mounted all bonded specimens on acrylic resin bases, we used a universal testing machine (Zwick GmbH, Ulm, Germany) to apply shear loading to the specimens, which used a knife-edge system at a $0.5 \mathrm{~mm} / \mathrm{min}$ crosshead speed until they fractured.

We divided the maximum load recorded upon failure (Newtons, N) to the bracket mesh area $\left(11.86 \mathrm{~mm}^{2}\right)$ to calculate the bond strength in MPa.

After debonding, close disc examination under $\times 40$ magnification was done to localize and report the site of the bond failure and the adhesive remnant index ARI. This index is calculated using the following scoring system:

1. The bracket imprint and all resin were retained on porcelain;

2. More than $90 \%$ of resin was retained on porcelain;

3. More than $10 \%$ but under $90 \%$ of resin was retained on porcelain;

4. Less than $10 \%$ of resin was retained on porcelain;

5. No resin on porcelain. ${ }^{18}$

The samples were immersed in glutaraldehyde solution of $2.5 \%$ for 12 hours at a temperature of $4^{\circ} \mathrm{C}$ for fixing; and after using distilled water to rinse the remnant, they were dehydrated in the ascending concentrations of ethanol (25\%: 20 minutes, 50\%: 20 minutes, 75\%: 20 minutes, 95\%: 30 minutes, 100\%: 60 minutes) for scanning electron microscope (SEM) analysis (DSM 960A, Zeiss, Germany). ${ }^{19}$ Then as the final step, we dried the samples using absorbent paper and sputter-coated with gold, and ultimately, we analyzed the surfaces utilizing a scanning electron microscope in a magnification of $\times 2000$ (Figure $1 \mathrm{~A}-\mathrm{C})$.

We used the Kolmogorov-Smirnov test to confirm normal data distribution. To compare SBS values among the groups, we used the Tukey's post hoc test and one-way ANOVA in SPSS 16.00 software (Microsoft, IL, USA). For ARI analysis, we used the Kruskal-Wallis test.

The threshold for significance for all statistical analyses was set at a probability value of 0.05 or less.

\section{Results}

Figure 1A-C demonstrates the SEM images of zirconia surfaces after surface treatment with HF acid-bur, sandblast, and Nd:YAG (1.5 W). 

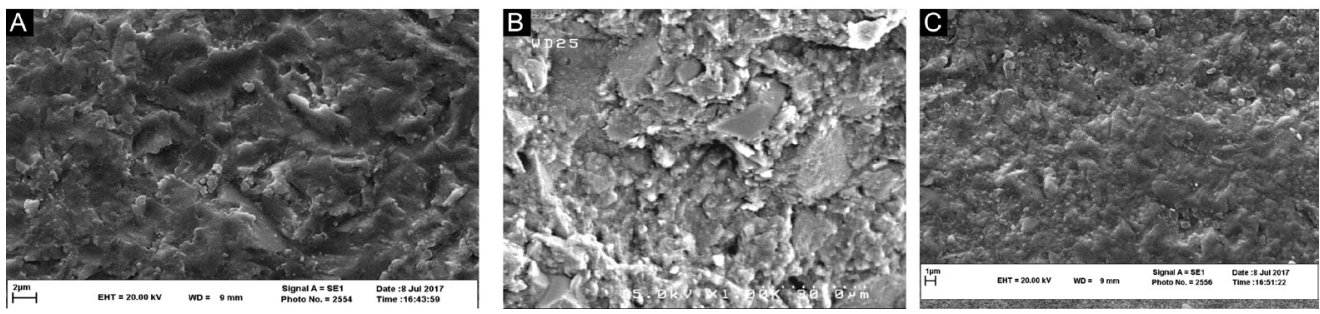

Figure 1. (A) SEM for the sample treated by HF. (B) SEM for the sample treated by the Nd:YAG laser. (C) SEM for the sample treated by sandblasting

The mean \pm SD of SBS in the HF group, the Laser group, and the sandblast group was $6.2 \pm 0.9 \mathrm{MPa}, 6 \pm 0.6 \mathrm{MPa}$, $3.1 \pm 0.8 \mathrm{MPa}$ respectively. The HF group demonstrated the highest SBS followed by the Nd:YAG and sandblasting groups.

Significant differences in the SBS of metal brackets to the zirconium surface were observed in the results of a one-way analysis of variance in different preparation methods $(P<0.001)$. On the other hand, in terms of the SBS of metal brackets to zirconia surfaces, the results of the paired comparison test showed a significant difference between the HF and laser groups and group $\mathrm{c}(P<0.001)$ and no significant difference between the HF group and the laser group $(P=0.03)$ (Figure 2).

The maximum values of the adhesive remnant on the zirconia disc were observed in the HF and Nd:YAG laser groups (due to a high frequency of indices 1 and 2). However, the degrees of the adhesive residue on disc surfaces in the sandblast group have been relatively smaller (a high frequency of indices 4 and 5) (Table 1).

There was no statistically significant difference among the three groups regarding ARI by using the nonparametric Kruskal-Wallis test $(P=0.059)$.

\section{Discussion}

Achieving a suitable bond between zirconia surfaces and orthodontic brackets has always been a challenge to

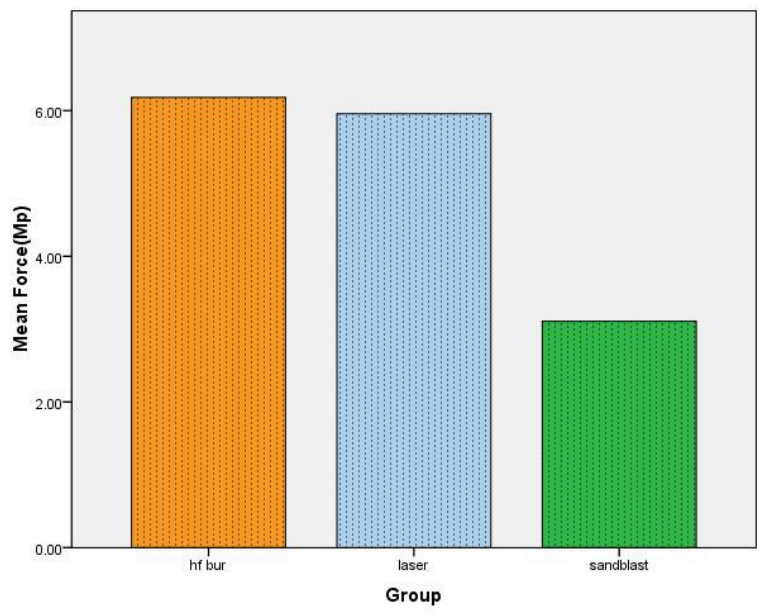

Figure 2. Comparison of Shear Bond Strength of 3 Groups. clinicians and researchers due to the importance of the exertion of orthodontic force without the debonding of brackets during the treatment. Considering the physical properties of the zirconia surface and the chemical characteristics of bonding resins, these surfaces are not suitable for the penetration of resin..$^{20}$ Commonly used surface treatment methods, are time-consuming or harmful to soft tissues, which is why even though HFA etching is a practical surface roughening method for bonding of porcelain ad composite; extreme caution is warranted as it is being applied in the oral cavity because there is a high risk of toxicity and soft tissue burns, which in turn makes many orthodontists hesitant to use this method. ${ }^{21,22}$ Therefore, achieving suitable surface treatments through the radiation of different types of laser has been proposed for this purpose. We conducted this study to examine the SBS of orthodontic metal brackets bonded to monolithic ceramic restoration treated by three different methods. Reynolds concluded that a bracket bond strength that is clinically ideal is between 5.8 and $7.8 \mathrm{MPa}{ }^{23}$ In this study, the SBS of the hydrofluoric acid and laser groups hit the 5.8 mark, while the SBS of the sandblast group was found to be less than $5.8 \mathrm{MPa}$.

Sandblasting by $110-\mu \mathrm{m}$ aluminum oxide particles achieved a clinically substandard SBS which was found to be lower than the acceptable range. As an alternative surface roughening option, we also investigated the reliability of Nd:YAG for bonding brackets to the zirconia surface with orthodontic adhesive.

Laser etching, also known as surface preparation by a laser, produces heat, which in turn causes porositties on the surface of the zirconia which provides mechanical retention for bonding.

Up until now few studies have evaluated bracket

Table 1. ARI Distribution in 3 Groups

\begin{tabular}{lllllll}
\hline & ARI & $\mathbf{1}$ & $\mathbf{2}$ & $\mathbf{3}$ & $\mathbf{4}$ & $\mathbf{5}$ \\
\hline HF & No. & 3 & 5 & 4 & 2 & 1 \\
& $\%$ & 20 & 33.3 & 26.7 & 13.3 & 6.7 \\
\multirow{2}{*}{ Laser } & No. & 2 & 3 & 4 & 5 & 1 \\
& $\%$ & 13.3 & 20 & 26.7 & 33.3 & 6.7 \\
Sandblast & No. & 0 & 2 & 6 & 3 & 4 \\
& $\%$ & 0 & 13.3 & 40 & 20 & 26.7 \\
\hline
\end{tabular}


bonding strength to the zirconia surface and no study has used the Nd-YAG laser for zirconia surface preparation before bracket bonding. Similar studies have been done on feldspathic porcelain crowns. For instance, in a study conducted by Hosseini et al, for measuring SBS of brackets, 72 feldspathoid porcelain samples were prepared and categorized into six groups: HF 9.6\%, Nd:YAG (0.75 W, $1 \mathrm{~W}, 1.25 \mathrm{~W}, 1.5 \mathrm{~W}, 2 \mathrm{~W})$. In contrast to the results obtained from the current study, they found $\mathrm{Nd}$ :YAG laser radiation of 1.5 and $2 \mathrm{~W}$ was acceptable regarding SBS. ${ }^{15}$ The result of the study conducted by Kim and Cho shows that the Nd:YAG laser has the ability to improve the SBS of the titanium porcelain interface..$^{20}$ Poosti et al showed that even though irradiation with 2 and $3 \mathrm{~W}$ powers of the Er:YAG laser did not result in acceptable bond strength, the $0.8 \mathrm{~W}$ power of $\mathrm{Nd}$ :YAG laser irradiation could result in satisfactory bond strength for clinical orthodontic practice. ${ }^{18}$ Akova et al believed that greater SBS was achieved in the Co2 laser-treated group in comparison with conventional methods such as $\mathrm{HF}$ acid etching. ${ }^{2}$ Yassaei et al compared the effect of the Er:YAG laser (power outputs of 1.6, 2 and $3 \mathrm{~W}$ ) and 9.6\% HF acid on the SBS of metal brackets and porcelain discs which resulted in an insignificant difference between the methods used. They did not assess sandblasting. ${ }^{\text {? }}$

Murthy et al also used five different surface treatments to prepare zirconium crowns for bonding to autopolymerizing resin. Their study showed that the $\mathrm{CO} 2$ laser resulted in the highest SBS after using HF acid etching and sandblasting with $110 \mu \mathrm{m}$ alumina. ${ }^{24} \mathrm{We}$ did not use the CO2 laser in our study and SBS using $\mathrm{HF}$ acid etching was higher than the Nd-YAG laser and sandblasting.

A comparison of the results of different studies in this area suggests some incongruence. It seems that the difference in study methods is the cause of these differences and sometimes inconsistent findings. It has been reported that structural changes of the samples, which are developed in response to radiation of lasers, are dependent on the intensity of laser energy, duration of radiation, and the distance between the radiation source and the sample surface. The Nd:YAG laser causes less surface degradation and thermogenesis due to a lower wavelength in comparison with Er:YAG and carbon dioxide lasers. ${ }^{22,25}$

The application of the Nd:YAG laser in comparison with HF etching reduces the preparation time significantly, (10 seconds versus 3-5 minutes).

Unlike the results obtained in this study regarding the greater bond strength of the group prepared with the laser in comparison with the sandblast group, in a study conducted by Arami et al on the attachment of composite resin to zirconium, they found that $\mathrm{Al}_{2} \mathrm{O}_{3}$ particles are the most efficient method of surface treatment. They also showed that the application of the Er-YAG laser (2 W) has a greater effect than the Nd:YAG laser $(1.5 \mathrm{~W}) .{ }^{26}$
Scanned micrograph images with $\times 2000$ magnification were obtained after completing the roughening procedure.

An analysis using a scanning electron microscope (SEM) was done after the samples were immersed in a $2.5 \%$ solution of glutaraldehyde for 12 hours at $4^{\circ} \mathrm{C}$ for fixing and then rinsed in distilled water. After that, they were dehydrated in the ascending concentrations of ethanol (25\%: 20 min, 50\%: 20 minutes, 75\%: 20 minutes, 95\%: 30 minutes, and 100\%: 60 minutes). The images prepared were qualitative, and they confirmed the development of relatively suitable porosity in the three groups visually. It seems that the sample prepared by HF 9\%-bur indicates greater superficial porosity followed by the laser group and the sandblast group respectively. It should also be noted that the evaluation of SEM images was qualitative, and many factors are interruptive. In addition, out of each group, only one sample was chosen for the qualitative assessment of the surface preparation method, which can also limit the accuracy of findings.

Based on the results of this research, over half of the samples (30 out of 45) had ARI of 3 and above, suggesting that debonding has occurred mainly in resin-ceramic contact areas. Clinically, this type of debonding has been reported to be more desirable, as in this state, there is less need to clear debonded zirconia, and thus the risk of surface damage will diminish. ${ }^{27,28}$ Under clinical conditions, the frequency of this type of debonding is also greater because under these conditions, the provision of a desirable etching on the zirconia surface is more difficult due to uncontrolled factors such as humidity, temperature, time, and movements of the patient. ${ }^{27}$ Furthermore, the structural pattern of the bracket base is such that debonding is not common at the resin-bracket contact area. $^{29}$

In contrast to the findings of this research, in evaluating bonded brackets, Lee et al showed that in the samples prepared by the etching acid method with Er:YAG laser radiation, the debonding was mainly of adhesive type at the resin-bracket contact area. ${ }^{30}$ This incongruence can be due to the difference in the manner of the debonding test, which in this study was performed by exerting a stretching force. Valletta et al showed that debonding under a stretching force may occur at the bracket-resin contact area while debonding under shear force mainly occurs at the resin-tooth contact area. ${ }^{31}$ In a research study by Fernandez and Canut that also performed a stretching strength test, debonding occurred mainly in the bracket-resin contact area. ${ }^{29}$ On the other hand, some researchers believe that debonding at the tooth/zirconia and composite area is not favorable as it can cause a cohesive breakdown in their structure and superficial degradation of detachment type. . $^{21,32}$

This research has been performed in vitro and its results should be interpreted in accordance to limitations of this type of study. Under experimental conditions, the forces exerted to brackets are different from the forces 
present under clinical conditions. In the mouth, brackets are influenced by different types of stretching, shear, rotational, and combined forces, and the forces that are applied for the removal of brackets in the clinic are different from the pure shear force that is applied gradually in a laboratory. In addition, in the oral cavity, a set of stresses including changes in temperature, humidity, acidity, and also microbial plaque exist, thereby complicating their simulation under experimental conditions. ${ }^{27,28}$ In spite of these limitations, the usage of experimental methods before the application of different materials under clinical conditions is the best and most suitable option.

\section{Conclusion}

The results of this research evaluating the SBS of metal brackets to zirconia following surface treatment indicated that the SBS was acceptable for clinical orthodontic practice in hydrofluoric acid and Nd:YAG groups. The minimal bond strength was in the sandblasting group. Evaluation of the effects of different powers of various lasers on the bond of metal brackets to zirconia can be suggested.

\section{Ethical Considerations}

Ethical approval was obtained from Mazandaran University ofMedical Sciences (IR.MAZUMS.REC.95.2591).

\section{Conflict of Interests}

The authors declare no conflict of interest.

\section{References}

1. Sobouti F, Rakhshan V, Chiniforush N, Khatami M. Effects of laser-assisted cosmetic smile lift gingivectomy on postoperative bleeding and pain in fixed orthodontic patients: a controlled clinical trial. Prog Orthod. 2014;15(1):66. doi: 10.1186/s40510-014-0066-5.

2. Akova T, Yoldas O, Toroglu MS, Uysal H. Porcelain surface treatment by laser for bracket-porcelain bonding. Am J Orthod Dentofacial Orthop. 2005;128(5):630-7. doi: 10.1016/j.ajodo.2004.02.021.

3. Rekow ED, Silva NR, Coelho PG, Zhang Y, Guess P, Thompson V. Performance of dental ceramics: challenges for improvements. J Dent Res. 2011;90(8):937-52. doi: 10.1177/0022034510391795.

4. Manicone PF, Iommetti PR, Raffaelli L. An overview of zirconia ceramics: basic properties and clinical applications. J Dent. 2007;35(11):819-26. doi: 10.1016/j. jdent.2007.07.008.

5. Visuri SR, Gilbert JL, Wright DD, Wigdor HA, Walsh Jr JT. Shear strength of composite bonded to Er:YAG laserprepared dentin. J Dent Res. 1996;75(1):599-605. doi: 10.1177/00220345960750011401.

6. Vijayaraghavan R, Rao VAP, Reddy NV, Krishnakumar R, Sugumaran DK, Mohan G. Assessment and comparison of microleakage of a fluoride-releasing sealant after acid etching and Er:YAG laser treatment - An in vitro study. Contemp Clin Dent. 2012;3(1):64-8. doi: 10.4103/0976237X.94549.
7. Yassaei S, Moradi F, Aghili H, Lotfi Kamran MH. Shear bond strength of orthodontic brackets bonded to porcelain following etching with Er:YAG laser versus hydrofluoric acid. Orthodontics (Chic). 2013;14(1):e82-7. doi: 10.11607/ ortho.856.

8. Raigrodski AJ, Chiche GJ, Potiket N, Hochstedler JL, Mohamed SE, Billiot S, et al. The efficacy of posterior three-unit zirconium-oxide-based ceramic fixed partial dental prostheses: A prospective clinical pilot study. J Prosthet Dent. 2006;96(4):237-44. doi: 10.1016/j. prosdent.2006.08.010.

9. Zhang Y, Chai H, Lee JJW, Lawn BR. Chipping resistance of graded zirconia ceramics for dental crowns. J Dent Res. 2012;91(3):311-5. doi: 10.1177/0022034511434356.

10. Sobouti F, Rakhshan V, Gholamrezaei Saravi M, Zamanian A, Shariati M. Two-year survival analysis of twisted wire fixed retainer versus spiral wire and fiber-reinforced composite retainers: a preliminary explorative single-blind randomized clinical trial. Korean J Orthod. 2016;46(2):10410. doi: 10.4041/kjod.2016.46.2.104.

11. Lee JY, Kim JS, Hwang CJ. Comparison of shear bond strength of orthodontic brackets using various zirconia primers. Korean J Orthod. 2015;45(4):164-70. doi: 10.4041/ kjod.2015.45.4.164.

12. Zachrisson YØ, Zachrisson BU, Büyükyilmaz T. Surface preparation for orthodontic bonding to porcelain. Am J Orthod Dentofacial Orthop. 1996;109(4):420-30. doi: 10.1016/S0889-5406(96)70124-5.

13. Blakey R, Mah J. Effects of surface conditioning on the shear bond strength of orthodontic brackets bonded to temporary polycarbonate crowns. Am J Orthod Dentofacial Orthop. 2010;138(1):72-8. doi: 10.1016/j.ajodo.2008.08.030.

14. Karan S, Büyükyılmaz T, Toroğlu MS. Orthodontic bonding to several ceramic surfaces: are there acceptable alternatives to conventional methods? Am J Orthod Dentofacial Orthop. 2007;132(2):144.e7-14. doi: 10.1016/j.ajodo.2006.12.006.

15. Hosseini MH, Sobouti F, Etemadi A, Chiniforush N, Shariati M. Shear bond strength of metal brackets to feldspathic porcelain treated by Nd:YAG laser and hydrofluoric acid. Lasers Med Sci. 2015;30(2):837-41. doi: 10.1007/s10103013-1458-3.

16. Heravi F, Moazzami SM, Dehghani M. Effects of different surface preparations on shear bond strength of orthodontic brackets to porcelain. J Calif Dent Assoc. 2010;38(11):794-9.

17. Mirhashemi A, Sharifi N, Moharrami M, Chiniforush $\mathrm{N}$. Evaluation of different types of lasers in surface conditioning of porcelains: a review article. J Lasers Med Sci. 2017;8(3):101-111. doi: 10.15171/jlms.2017.19.

18. Poosti M, Jahanbin A, Mahdavi P, Mehrnoush S. Porcelain conditioning with Nd:YAG and Er:YAG laser for bracket bonding in orthodontics. Lasers Med Sci. 2012;27(2):321-4. doi: 10.1007/s10103-010-0878-6.

19. Hosseini MH, Sobouti F, Etemadi A, Chiniforush N, Bouraima SA. Scanning electron microscope comparative evaluation of feldspathic porcelain surfaces under irradiation by different powers of Neodymium-doped Yttrium Aluminium Garnet (Nd:YAG) laser. J Lasers Med Sci. 2013;4(2):75-8.

20. Paranhos MP, Burnett LH Jr, Magne P. Effect Of Nd:YAG laser and $\mathrm{CO}_{2}$ laser treatment on the resin bond strength to zirconia ceramic. Quintessence Int. 2011;42(1):79-89. 
21. Kim JT, Cho SA. The effects of laser etching on shear bond strength at the titanium ceramic interface. J Prosthet Dent. 2009;101(2):101-106. doi: 10.1016/S0022-3913(09)600020.

22. Barbosa VL, Almeida MA, Chevitarese O, Keith O. Direct bonding to porcelain. Am J Orthod Dentofacial Orthop. 1995;107(2):159-164. doi: 10.1016/s0889-5406(95)701311.

23. Reynolds IR, von Fraunhofer JA. Direct bonding of orthodontic brackets-a comparative study of adhesives. Br J Orthod. 1976;3(3):143-146. doi: 10.1179/bjo.3.3.143.

24. Murthy V, Manoharan, Balaji, Livingstone D. Effect of four surface treatment methods on the shear bond strength of resin cement to zirconia ceramics- a comparative in vitro study. J Clin Diagn Res. 2014;8(9):ZC65-8. doi: 10.7860/ JCDR/2014/10104.4872.

25. Kimura Y, Wilder-Smith P, Matsumoto K. Lasers in endodontics: a review. Int Endod J. 2000;33(3):173-85. doi: 10.1046/j.1365-2591.2000.00280.x.

26. Arami S, Tabatabae MH, Namdar SF, Chiniforush N. Effects of different lasers and particle abrasion on surface characteristics of zirconia ceramics. J Dent (Tehran). 2014;11(2):233-41

27. Ersu B, Yuzugullu B, Yazici AR, Canay S. Surface roughness and bond strengths of glass-infiltrated alumina-ceramics prepared using various surface treatments. J Dent. 2009;37(11):848-56. doi: 10.1016/j.jdent.2009.06.017.

28. Sinha PK, Nanda RS, Duncanson MG, Hosier MJ. Bond strengths and remnant adhesive resin on debonding for orthodontic bonding techniques. Am J Orthod Dentofacial Orthop. 1995;108(3):302-7. doi: 10.1016/s08895406(95)70025-0.

29. Fernandez L, Canut JA. In vitro comparison of the retention capacity of new aesthetic brackets. Eur J Orthod. 1999;21(1):71-7. doi: 10.1093/ejo/21.1.71.

30. Lee BS, Hsieh TT, Lee YL, Lan WH, Hsu YJ, Wen PH, et al. Bond strengths of orthodontic bracket after acid-etched, Er:YAG laser-irradiated and combined treatment on enamel surface. Angle Orthod. 2003;73(5):565-70.

31. Valletta R, Prisco D, De Santis R, Ambrosio L, Martina R. Evaluation of the debonding strength of orthodontic brackets using three different bonding systems. Eur J Orthod. 2007;29(6):571-7. doi: 10.1093/ejo/cjm056.

32. Schmage P, Nergiz I, Herrmann W, Özcan M. Influence of various surface-conditioning methods on the bond strength of metal brackets to ceramic surfaces. Am J Orthod Dentofacial Orthop. 2003;123(5):540-6. doi: 10.1067/ mod.2003.S0889540602569110. 